\title{
Erratum: Growth of stochastic resonance in neuronal ensembles with the input signal intensity [Phys. Rev. E 86, 011922 (2012)]
}

Shogo Yonekura, Yasuo Kuniyoshi, and Yoichiro Kawaguchi

(Received 9 March 2016; published 28 March 2016)

DOI: 10.1103/PhysRevE.93.039903

The effective noise intensity $D$ that is used to evaluate theoretical value of $\left\langle C_{1}\right\rangle$ throughout the original paper is inaccurate. In Sec. III A, we derived the piecewise-linear FitzHugh-Nagumo (plFHN) neuron model, and we introduced the noise scaling $\xi(t) \rightarrow 7 \tau \xi(\tilde{t})$ in correspondence with the time scaling $t \rightarrow 7 \tau \tilde{t}$. However, the noise should be scaled as $\xi(t) \rightarrow \sqrt{7 \tau} \xi(\tilde{t})$. Therefore, the plFHN neuron mode I in Eq. (6) and (A1) should read

$$
\begin{aligned}
\tilde{\epsilon} \dot{v}_{i}(\tilde{t}) & =\frac{1}{6} f\left(v_{i}\right)-\frac{\gamma}{6} w_{i}+2 \sqrt{3}\left(b-\frac{1}{2}\right) \\
\tau \dot{w}_{i}(\tilde{t}) & =\frac{6}{7 \gamma} v_{i}-\frac{1}{7} w_{i}+\frac{12 \sqrt{3}}{7 \gamma} g_{0} \sum_{j}^{N_{j}}\left(1+\tilde{\omega}_{j}\right) e^{\tilde{\omega}_{j} \tilde{t}}+\frac{12 \sqrt{3}}{7 \gamma} \sqrt{2 D_{0}} \xi_{i}(\tilde{t}) \sqrt{7 \tau}
\end{aligned}
$$

(a)

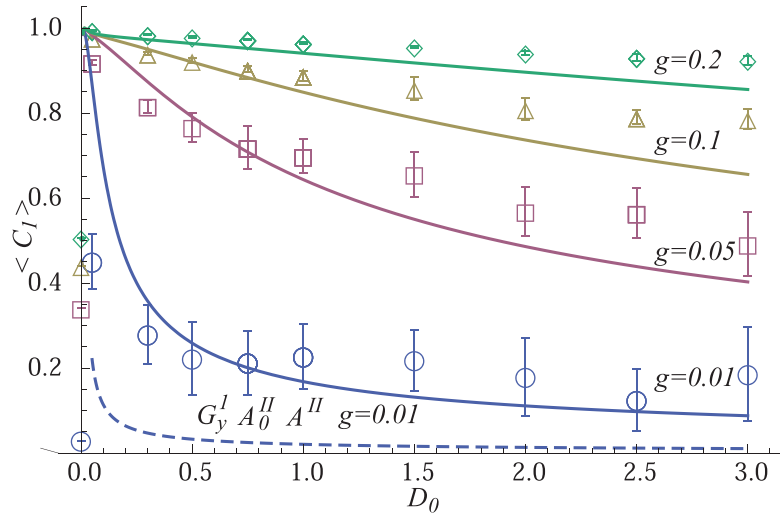

(c)

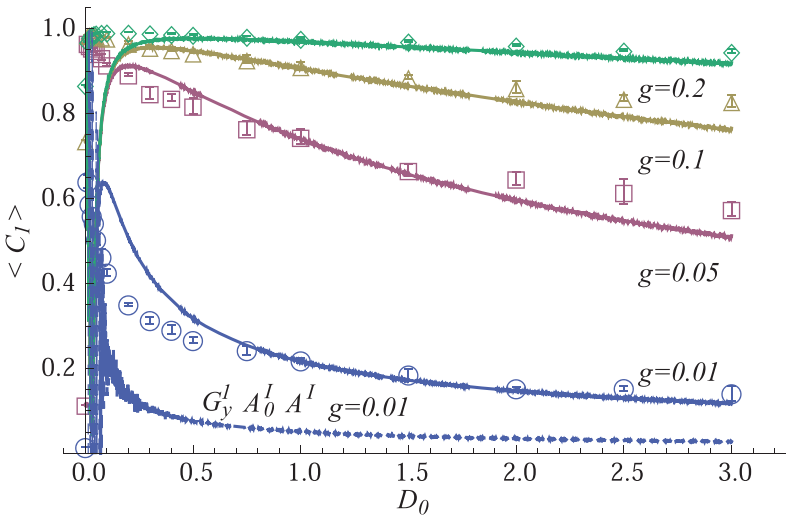

(b)

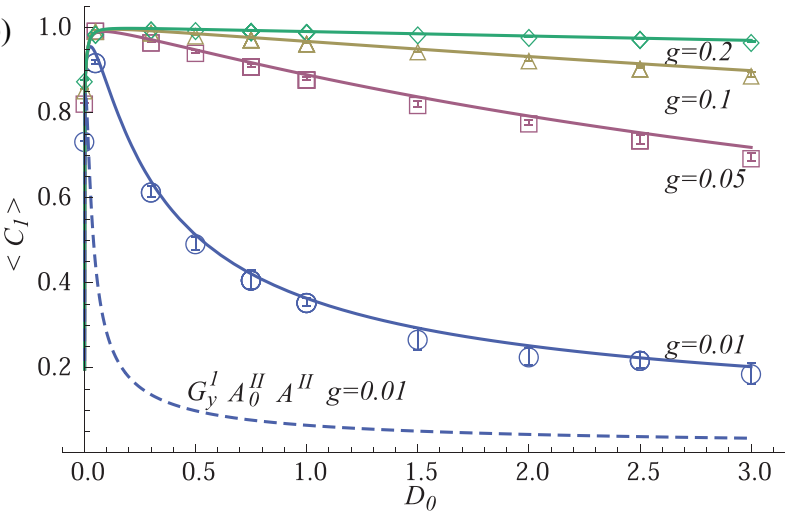

(d)

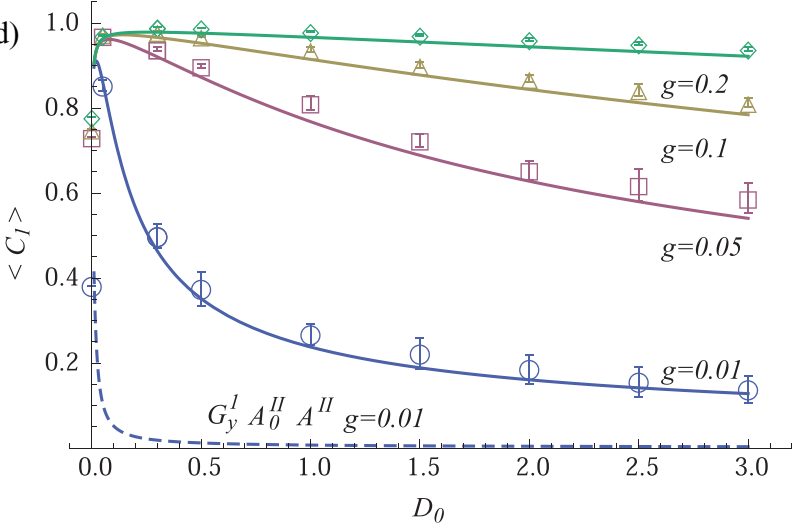

FIG. 1. The correlation coefficient $\left\langle C_{1}\right\rangle$ of the neuronal ensemble consisting of $N=2000$ neurons with a bias signal $b=0.3$, as a function of the noise intensity $D_{0}$ for (a)-(c) a periodic input signal and (d) a semiperiodic input and several input gains $g ; g=0.01 ; 0.05 ; 0.1 ;$ and 0.2 . The theoretical curves of $\left\langle C_{1}\right\rangle$ with $\sigma(D)^{2}=4 \pi R_{0}\left(1-R_{0}\right)$. The solid curves, in (a) are $\omega=0.1 \mathrm{~Hz}$ using the mode $\left(G_{y}^{1}, A_{0}^{I}, A^{I}\right)$; in (b), $\omega=1 \mathrm{~Hz}$ using $\left(G_{y}^{1}, A_{0}^{I}, A^{I}\right)$; in (c), $\omega=10 \mathrm{~Hz}$ using $\left(G_{y}^{2}, A_{0}^{I I}, A^{I I}\right)$; and in (d), $\omega_{1}=1$ and $\omega_{2}=\sqrt{2} \mathrm{~Hz}$ using $\left(G_{y}^{1}, A_{0}^{I}, A^{I}\right)$. The theoretical prediction of $\left\langle C_{1}\right\rangle$ using another signal and linear response mode pair with $g=0.01$ is shown for reference by the dashed lines. The numerical $\left\langle C_{1}\right\rangle$ values are obtained from 20 trials of a $100 \mathrm{~s}$ simulation and are represented by the symbols and error bars for $g=0.01(\bigcirc), 0.05$ ( $\square$ ), 0.1 $(\triangle)$, and $0.2(\diamond)$. The theoretical and numerical phase shift $\alpha$ are determined such that the result $C_{1}$ is a maximum. In all cases shown here, an increase in $g$ leads to growth of the SR profiles from bell-shaped curves into trapezoidally-shaped curves. 
Likewise, the plFHN neuron mode II in Eq. (A6) should read

$$
\begin{aligned}
& \tilde{\epsilon} \dot{v}_{i}=-\frac{1}{2} f\left(v_{i}\right)-\frac{\gamma}{6} w_{i}+2 \sqrt{3}\left(b+g_{0} \sum_{j}^{N_{j}} e^{\tilde{\omega}_{j} \tilde{t}}-\frac{1}{2}\right), \\
& \tau \dot{w}_{i}=\frac{6}{7 \gamma} v_{i}-\frac{1}{7} w_{i}+\frac{12 \sqrt{3}}{7 \gamma} \sqrt{2 D_{0}} \xi_{i}(\tilde{t}) \sqrt{7 \tau}
\end{aligned}
$$

The effective noise intensity $D$, defined in the third paragraph of Sec. III A, the first paragraph of Sec. III B, the third paragraph of Appendix A1, and the fourth paragraph of Appendix A2, should read

$$
D=\left(\frac{12 \sqrt{3}}{\gamma \sqrt{7 \tau}}\right)^{2} D_{0} .
$$

Furthermore, we used an incorrect assumption regarding $\sigma(D)^{2}$, the variance of the noise component $\eta(t)$ in Eq. (29). We assumed that $R_{y, i}^{1,2}(t)$ is a Poisson process and therefore $\sigma(D)^{2}=R_{0}$ (described in the second paragraph of Sec. III E and the caption of Fig. 1). However, plFHN neuron model is a two-state process [1] of $R_{y, i}^{1,2}=0$ or 1 , and the $\sigma(D)^{2}$ should read

$$
\sigma(D)^{2}=4 \pi \frac{\left\langle T_{x}\right\rangle\left\langle T_{y}\right\rangle}{\left(\left\langle T_{x}\right\rangle+\left\langle T_{y}\right\rangle\right)^{2}}=4 \pi R_{0}\left(1-R_{0}\right),
$$

where $T_{z}$ is the first passage time in either left or right branch, and $T_{z}=R_{z} / J_{z}$ ( $z$ is either $x$ or $y$ ). Using these corrected effective noise intensity $D$ and the variance $\sigma(D)^{2}$, Figs. 1-4 are corrected as Figs. 1-4, respectively.

The implication of these corrections is mainly observed in Fig. 2. First, in our previous paper, the theoretical value of $\left\langle C_{1}\right\rangle$ rapidly increases with the increase in $D_{0}$, whereas the numerical data shows a very slow increase in $\left\langle C_{1}\right\rangle$ for large negative and positive values of $b$, i.e., $b=-1$ (a) and $b=2(\mathrm{~d})$. This mismatch is resolved by introducing the correct dynamics of $\sigma(D)^{2}$

(a)

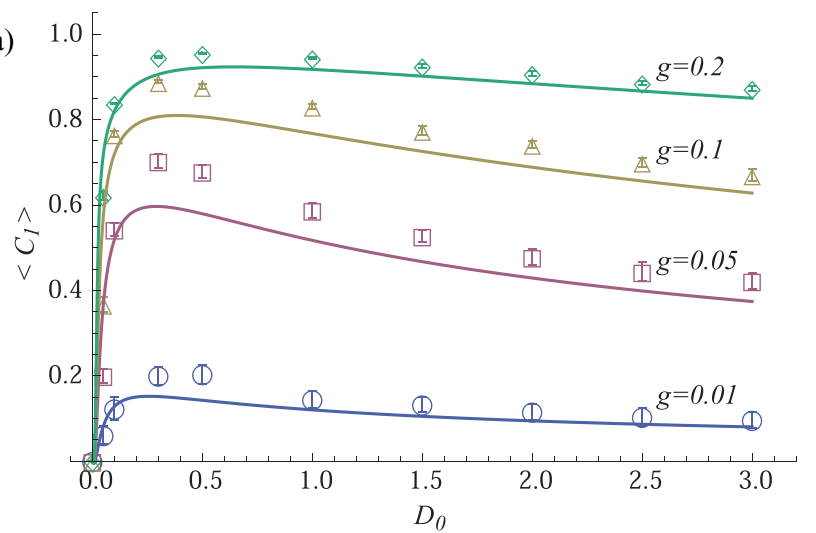

(c)

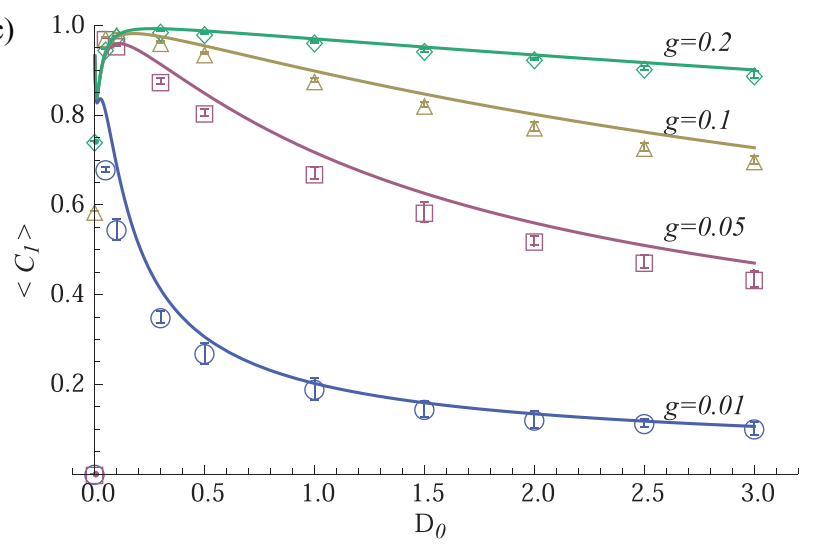

(b)

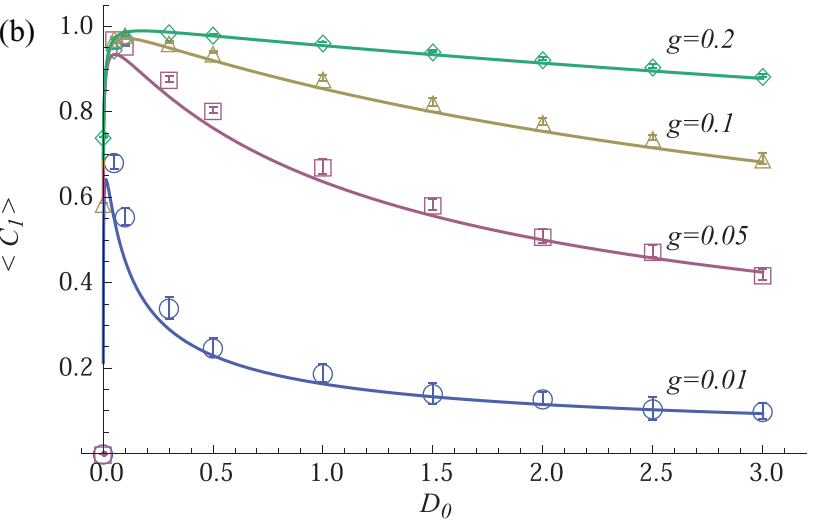

(d) 1

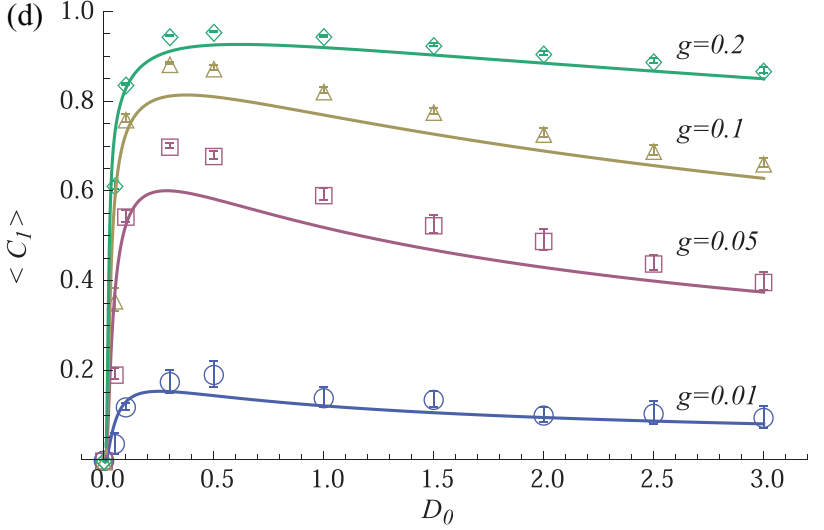

FIG. 2. The coefficient $\left\langle C_{1}\right\rangle$ in the mode $\left(G_{y}^{1}, A_{0}^{I I}, A^{I I}\right)$ in response to the periodic input signal of $\omega=1 \mathrm{~Hz}$ as a function of noise intensity $D_{0}$ with bias intensities of $b=$ (a) -1 , (b) 0 , (c) 1 , and (d) 2 . Note that biases $b=-1$ and 0 correspond to the subthreshold regime and that biases $b=1$ and 2 correspond to the excitation-blocked regime. Numerically obtained $\left\langle C_{1}\right\rangle$ are represented by the symbols and error bars as used in Fig. 1. The size of the neuron ensemble is $N=500$. It is clear that the growth of SR occurs also in the excitatory and the excitation-blocked firing regimes. 
(a)

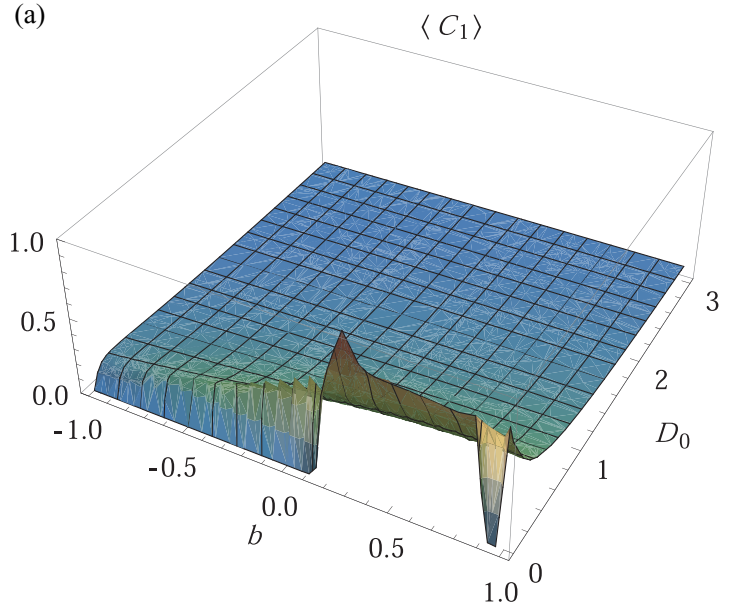

(c)

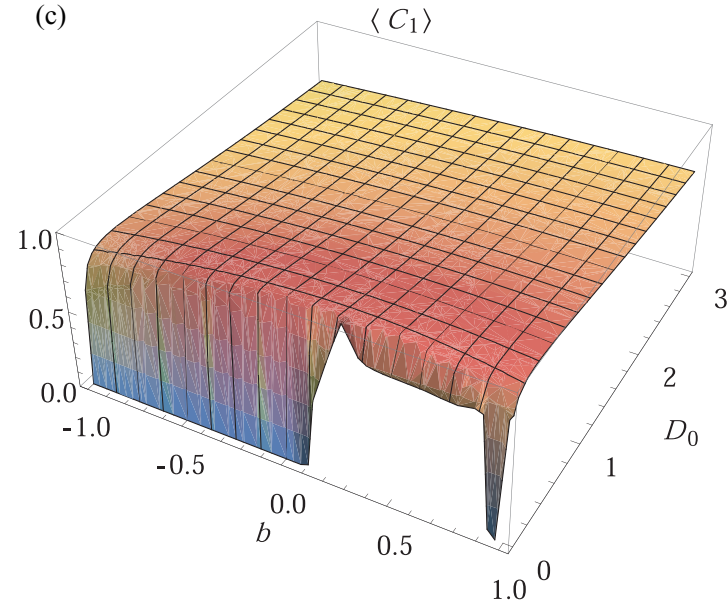

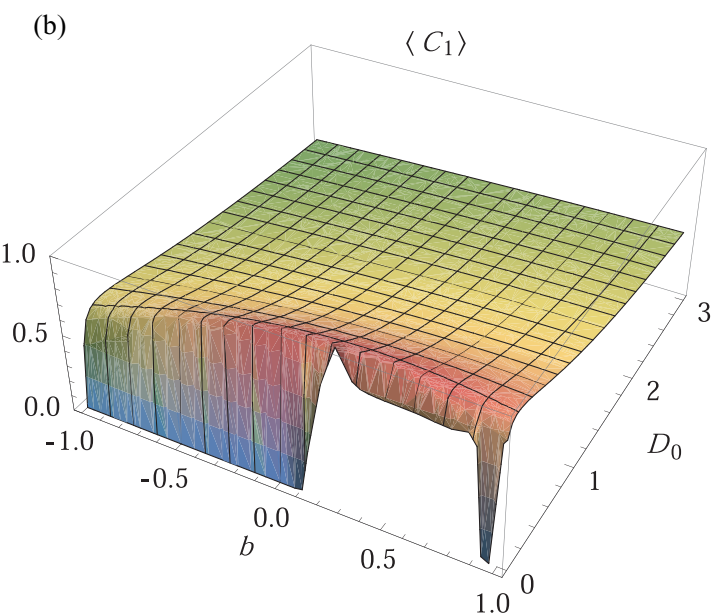

(d)

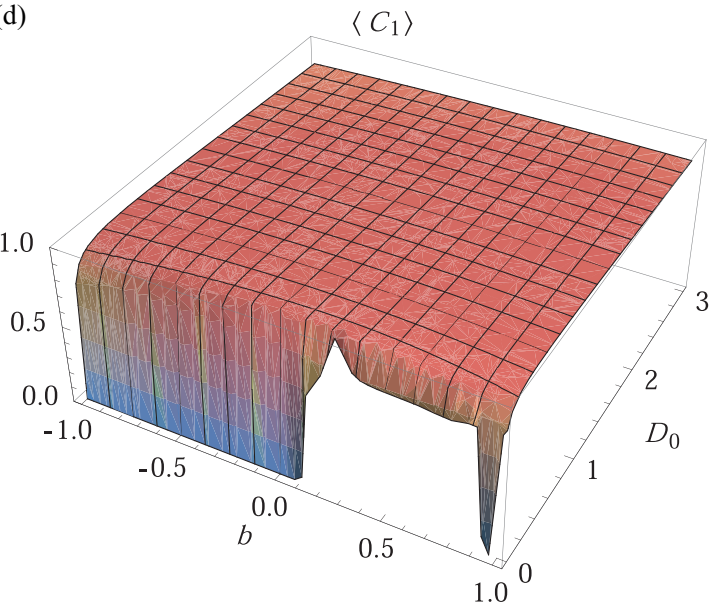

FIG. 3. Theoretical $\left\langle C_{1}\right\rangle$ of the $N=500$ neuron ensemble in the $\left(G_{y}^{1}, A_{0}^{I I}, A^{I I}\right)$ mode as a function of the noise intensity $D_{0}$ and bias signal $b$ for signal frequency $\omega=1 \mathrm{~Hz}$ and several input gains $g$. The meshes in (a) is $g=0.01$; in (b), $g=0.05$; in (c), $g=0.1$; and in (d), $g=0.2$. The steep, small peak in $\left\langle C_{1}\right\rangle$ for an input gain of $g=0.01$ grows larger with an increase in the signal intensity, leading to the emergence of SR without tuning. Note that the theoretical $\left\langle C_{1}\right\rangle$ for a relatively large $D_{0}$, i.e., $D_{0}>0.1$, is only weakly sensitive to changes in the bias signal $b$, although the bias $b$ is a dominant parameter of $\left\langle C_{1}\right\rangle$ for $D_{0}<0.1$.

in Eqs. (D1) and (D2). The slow increase of $\left\langle C_{1}\right\rangle$ with the increase in $D_{0}$ for large negative and positive value of $b$ can also be observed in Fig. 3.

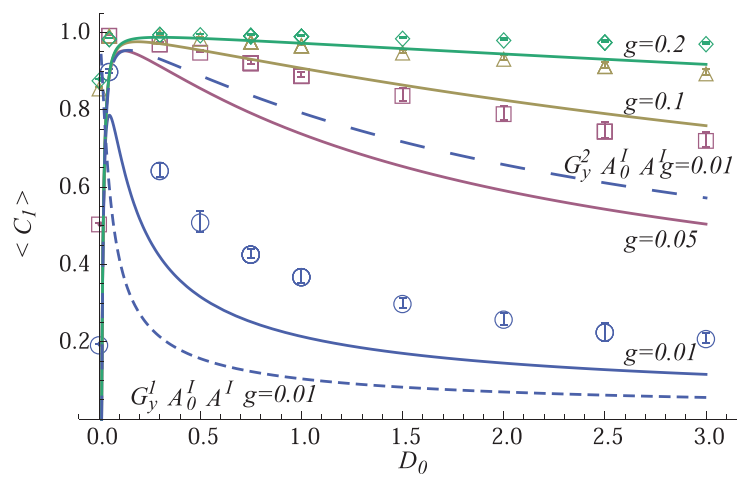

FIG. 4. The coefficient $\left\langle C_{1}\right\rangle$ in response to a periodic input signal of $\omega=5 \mathrm{~Hz}$ and bias $b=0.3$ as a function of the noise intensity $D_{0}$. The theoretical estimation of $\left\langle C_{1}\right\rangle$ in response to a signal frequency $\omega=5 \mathrm{~Hz}$ using the mode $G_{y}^{2}, A_{0}^{I I}$, and $A^{I I}$ with $g=0.01 ; 0.05 ; 0.1$; and 0.2 is shown by the solid lines, that using the mode $G_{y}^{1}, A_{0}^{I}$, and $A^{I}$ with $g=0.01$ is shown by the dashed line, and that using the mode $G_{y}^{2}$, $A_{0}^{I}$, and $A^{I}$ with $g=0.01$ is shown by the long dashed line. The theoretical $\left\langle C_{1}\right\rangle$ using the $G_{y}^{1}, A_{0}^{I I}$, and $A^{I I}$ mode is not shown as it falls well below the dashed line. Around the input frequency $\omega=5 \mathrm{~Hz}$, no single mode matches the numerical result. Numerically obtained $\left\langle C_{1}\right\rangle$ are represented by the same symbols and error bars as used in Figs. 1 and 2. The size of the neuron ensemble is $N=2000$. 
Secondly, the theoretical estimations of $\left\langle C_{1}\right\rangle$ for $D>1$ with bias $b=-1,2$ shown in Fig. 2(a) and 2(d) are slightly smaller than the numerical values of $\left\langle C_{1}\right\rangle$. This implies that the plFHN model oversimplifies the original cubic null cline dynamics of the FHN neuron model for large positive and negative bias signal.

In short, our results are qualitatively and quantitatively almost unchanged, and therefore the conclusions of the original paper are still valid.

Furthermore, we would like to correct a typographical error. Equation (13) should read

$$
\begin{aligned}
& \phi(x)=\frac{J_{0}}{D} e^{-x^{2} /(2 D)} \int_{x_{-}}^{x} d z e^{z^{2} /(2 D)} \Theta\left(x_{+}-z\right), \\
& \phi(y)=\frac{J_{0}}{D} e^{-y^{2} /(2 D)} \int_{y}^{y_{-}} d z e^{z^{2} /(2 D)} \Theta\left(z-y_{+}\right) .
\end{aligned}
$$

[1] B. Lindner, Coherence and Stochastic Resonance in Nonlinear Dynamical Systems, Ph.D. thesis, Humboldt-Universität zu Berlin, 2002. 